\title{
Imagen de Hugo Chávez y su gobierno: Tomás Eloy Martínez, Mario Vargas Llosa y Carlos Fuentes
}

\section{Antonio Casella*}

\section{Recibido: febrero de 2010 / Aceptado: septiembre de 2015}

El 2002 puede ser considerado un año crítico en la vida política venezolana. La promulgación inconsulta de más de 40 leyes por parte del Poder Ejecutivo a finales del año 2001 inició una sucesión de hechos que concluyeron en una huelga general que se inició en diciembre de 2002 y se suspendió al año siguiente. Estos hechos produjeron opiniones en todo el mundo. Entre tales opiniones encontramos la de tres renombrados escritores latinoamericanos: Tomás Eloy Martínez, Mario Vargas Llosa y Carlos Fuentes. El presente trabajo intenta reconstruir a partir del análisis semántico - pragmático la imagen que estos escritores poseían de Hugo Chávez y de su gobierno para ese momento. El corpus analizado lo constituyen el artículo de opinión La soga al cuello, del primer escritor mencionado; la charla titulada ¿Por qué fracasa América Latina? dictada por Vargas Llosa y una declaración de prensa ofrecida por Carlos Fuentes en Barcelona España y que el periodista tituló Carlos Fuentes no aboga por una salida militar. Estos textos fueron producidos en tres momentos diferentes de ese año y por lo tanto responden de una u otra forma a los momentos específicos en los que se produjeron.

Palabras clave: Escritores latinoamericanos / análisis semántico - pragmático / Hugo Chávez / gobierno / imagen 


\section{Introducción}

El año 2002 venezolano comenzó en medio de protestas y concluyó con una huelga general. En abril, tras incontables manifestaciones en su contra, el presidente, según palabras del General Lucas Rincón, quien para la fecha fungía de Jefe del Estado Mayor, renuncia a su cargo para luego ser restituido apenas dos días después. Mucho material escrito acerca de esos sucesos se produjo durante todo el año y, por supuesto, se ha producido posteriormente. Personalidades y organizaciones de diversos órdenes tanto de Venezuela como del mundo, expresaron y continúan expresando sus apreciaciones acerca esos eventos.

En el presente estudio se intenta reconstruir la imagen que de Hugo Chávez y su gobierno expresaron tres escritores latinoamericanos durante ese año. Se trata de Tomás Eloy Martínez, Mario Vargas Llosa y Carlos Fuentes quienes expusieron al mundo sus pareceres acerca de lo que se vivía en este país en esos momentos. En tiempos no coincidentes, a través de diferentes instrumentos y dispositivos emitieron sus opiniones sobre Venezuela y su presidente.

Desde el punto de vista metodológico, el trabajo se ubica en el enfoque semántico pragmático del análisis del discurso. Se basa teóricamente en la propuesta que, a partir de Pottier (1992), Charaudeau, Van Dijk (1977) y Adam, entre otros, los estudiosos Molero y Cabeza (2005) han sistematizado. El análisis realizado es de carácter comparativo en la medida que se establecen puntos de encuentro y de divergencia entre los tres autores.

\section{Corpus y contexto social}

Venezuela adquirió para el mundo relevancia noticiosa a partir de la asunción de Hugo Chávez a la Presidencia de la República. La promesa de adelantar una revolución, convertida en socialista a partir de $2005^{1}$, las reiteradas expresiones anti norteamericanas, el acercamiento a grupos de la izquierda radical latinoamericana, las visitas a jefes de estado como Hussein y Gadafi y su estrecha relación con Fidel Castro propiciaron una mayor atención a los acontecimientos venezolanos.

En diciembre de 2001 la principal asociación venezolana de empresarios, FEDECAMARAS, en protesta por la promulgación de mas de 40 leyes por parte del poder ejecutivo que había sido habilitado por el parlamento para dictar leyes, convocó a un paro cívico de un día. A partir de ese momento, la situación interna se hizo cada vez más tensa hasta desembocar en la renuncia del Presidente el 11 de abril, su posterior restitución en el cargo dos días más tarde y en el paro general de finales del 2002.

Ante los múltiples sucesos del 2002 se ha producido una infinidad de opiniones, estudios, recomendaciones, etc. de una igual infinidad de personalidades y organizaciones. Unos, emitidos durante los diversos acontecimientos y otros, más sosegados, posteriormente.

1 El llamado permanente a la confrontación de clases, la violencia como posibilidad, el ataque a la democracia representativa por su formalidad y otros temas indican la presencia del marxismo en el discurso chavista. 
El corpus del trabajo está constituido por lo escrito $\left(\right.$ discurso ${ }^{2}$ ) por los tres escritores latinoamericanos mencionados. El primero es un artículo de prensa, titulado La soga al cuello, de Tomás Eloy Martínez ${ }^{3}$, publicado en El País de España y en La Nación de Argentina, en éste fue publicado el mes de febrero del año 2002, en plena efervescencia política y pocos días antes de la renuncia del presidente.

El segundo es un texto titulado ¿Por qué fracasa América Latina?, basado en un discurso de Mario Vargas Llosa ${ }^{4}$, dado en octubre del 2002 durante el lanzamiento de la Fundación Internacional para la Libertad en Madrid. Este texto fue publicado originalmente en enero/febrero de 2003 en inglés en el Cato Policy Report y fue reproducido en la Revista Inter-Forum del 7 de febrero de 2003, de donde lo hemos tomado. Este discurso se produjo unos meses después de la vuelta de Hugo Chávez a la Presidencia y pocos días antes de declararse la huelga general. La situación durante este mes de octubre se podría decir que era tan tensa como la de los primeros meses del año.

El tercer documento analizado es una declaración de prensa ofrecida el 13 de diciembre de 2002 por Carlos Fuentes ${ }^{5}$, desde Barcelona, España en ocasión de su participación en la Fiesta de la Literatura Kosmópolis organizada por el Centro de Cultura Contemporánea de Barcelona. Publicada en el Nacional de Caracas el día 14/12/2002 y titulada por el periodista Carlos Fuentes no aboga por una salida militar. Ya para esta fecha había comenzado el paro general y la declaración viene a ser una propuesta para salir de la crisis que se vivía en el momento.

El texto de Tomás Eloy Martínez (2002) es el único que trata exclusivamente sobre Venezuela, su presidente y el gobierno. Los otros dos no. El de Vargas Llosa, ofrece un conjunto de ideas acerca del fracaso de América Latina en torno al desarrollo y menciona a Hugo Chávez y su gobierno como ejemplo de lo negativo que acontece en América Latina. El texto de Carlos Fuentes es una nota periodística basada en una entrevista en la que el escritor hace referencia a tópicos diversos entre los cuales se encuentra Venezuela.

2 Se entiende por discurso, siguiendo a Molero (2003), además de "una unidad lingüística" "una práctica social que expresa o refleja entidades, comportamientos y relaciones y que además los constituye y conforma"

3 Tomás Eloy Martínez, es Argentino, escritor, ensayista y crítico literario, ganador del premio Alfaguara 2002, su obra ha sido considerada una de las más sólida de la literatura latinoamericana.

4 Mario Vargas Llosa, es peruano, escritor, ganador de una diversidad de premios literarios, entre ellos el premio venezolano Rómulo Gallegos de Novela, por su novela Casas Verde. Fue candidato presidencial en Perú frente a Fujimori. Es un crítico importante del estatismo en todas sus versiones, de las formas dictatoriales del poder y un exponente mundial de las ideas liberales.

5 Carlos Fuentes, es mexicano, novelista, ganador de una multiplicidad de premios literarios y, al igual que Vargas Llosa, del premio Rómulo Gallegos por su novela Terra Nostra. Se autodenomina de Izquierda, ha dicho, por ejemplo: "La izquierda siempre representa una posibilidad de desarrollo con mayor humanismo, representa el único techo político con el que cuentan los más desprotegidos... Por eso yo me considero de izquierda" (Fuentes, 2002) 


\section{Objetivos}

El trabajo reconstruye la imagen que del presidente Hugo Chávez y su gobierno poseían para el año 2002, Tomás Eloy Martínez, Mario Vargas Llosa y Carlos Fuentes.

En primer lugar, se identificaron los temas o tópicos que sintetizan los núcleos de significado de los discursos estudiados y que sirven para la construcción de la imagen de Hugo Chávez y su gobierno.

En segundo lugar, en el nivel lingüístico, se identifican los ámbitos o dominios desde los cuales se representa la imagen de Hugo Chávez y su gobierno.

En tercer lugar, en el mismo nivel se identifican los campos léxicos de construcción de la imagen de Hugo Chávez y de su gobierno.

Y en cuarto lugar, se determinan los diversos modos de organización del discurso a través de los cuales se construye la imagen de Hugo Chávez y su gobierno.

\section{Nivel lógico conceptual}

El discurso posee dos componentes (Molero, 2003). El pragmático, que tiene que ver con lo interactivo del discurso, con los participantes y con el contexto en el cual se produce. Y el semántico, que está referido a la información que se encuentra en el discurso; son los significados que se transmiten a los receptores; es lo que se dice y el cómo se dice. Es sobre este último componente que versa primordialmente el trabajo.

La metodología propuesta por Molero y Cabeza (2005) contempla cuatro niveles: el nivel referencial, el nivel conceptual, el nivel lingüístico y el nivel del discurso y se recorre en el análisis semasiológicamente, es decir que sube del nivel del discurso hasta los niveles lingüístico y lógico conceptual (Molero, 2003; 1985; 1999; 2002).

Al nivel que nos ocupa, el lógico conceptual, pertenecen las conceptuaciones de los eventos de los cuales el discurso da cuenta. De allí que se construya en primer lugar lo que los autores Molero y Cabeza (2005) denominan el Esquema Básico Incluyente de cada uno de los textos en cuestión.

\subsection{Esquema Básico Incluyente (EBI)}

El EBI es uno o más enunciados que pueden considerarse el epítome, lo esencial del discurso que se analiza, una síntesis. El conocimiento de éste se logra a través de un proceso o recorrido analítico que va de las micro-estructuras del discurso pasando por los temas, hasta llegar a la macro estructura. De allí que establecer el EBI sea un proceso de reducción textual que presenta la información contenida en el discurso en su nivel más general.

El artículo de opinión de Tomás Eloy Martínez fue escrito especialmente para dar cuenta del caso venezolano y está compuesto de 13 párrafos. El discurso de Vargas Llosa consta de 18 párrafos y se refiere directamente al Presidente Chávez y a su gobierno en los párrafos 5 y 12; sin embargo, el resto del discurso toca de una u otra forma al caso Venezolano. Por último, la declaración de prensa de Carlos Fuentes 
se distribuye en 16 párrafos, de los cuales 6 (del 11 al 16) están dedicados a asuntos literarios de allí que no se haga referencia a ellos; asimismo, se ha prescindido de aquellas frases u opiniones que el periodista-entrevistador agrega y que dan forma a la noticia; se centra, el trabajo, solo en lo expresado por Carlos Fuentes acerca de la situación venezolana y de su presidente.

Sin entrar a detallar cada una de las micro-estructuras, se muestran a continuación los tópicos o temas tratados por cada uno de los autores, de manera tal que el lector adquirirá una primera y genérica visión de los documentos mencionados:

Cuadro 1. Temas desarrollados por los tres autores

\begin{tabular}{|l|l|l|}
\hline Temas & Temas & Temas \\
\hline Tomás Eloy Martínez & Mario Vargas Llosa & Carlos Fuentes \\
\hline $\begin{array}{l}\text { Venezuela no sabe como } \\
\text { salir de Chávez }\end{array}$ & Desarrollo integral & Mal gobierno \\
\hline $\begin{array}{l}\text { La salida debe ser } \\
\text { democrática pero se } \\
\text { corren peligros }\end{array}$ & $\begin{array}{l}\text { América Latina no hay } \\
\text { confianza en Instituciones }\end{array}$ & Mal gobernante \\
\hline Mal gobierno & $\begin{array}{l}\text { Política Latinoamericana } \\
\text { dominada por la corrupción }\end{array}$ & $\begin{array}{l}\text { Salida a crisis debe ser } \\
\text { democrática y venezolana y } \\
\text { con ayuda latinoamericana }\end{array}$ \\
\hline $\begin{array}{l}\text { Chávez no tiene talento } \\
\text { ni decisión }\end{array}$ & $\begin{array}{l}\text { Chávez ejemplo del fracaso } \\
\text { latinoamericano. Mal gobierno }\end{array}$ & Chávez dictador \\
\hline Elección de Chávez & Elección de Chávez & Cambio Político \\
\hline Régimen autoritario. & Necesidad de políticas liberales & \\
\hline
\end{tabular}

En su artículo, Tomás Eloy Martínez dirige primero la mirada hacia la "tragedia” que vive Venezuela: eligió democráticamente a Hugo Chávez que posee una serie de características indeseables y ahora (2002) no sabe cómo salir de él. Y segundo, realiza una alerta acerca de los peligros de adelantar los tiempos democráticos y señala que si "los gobernantes se cambiaran al azar de su popularidad, en el mundo entero se viviría un minué político de vértigo en el que prosperarían los demagogos." Acepta la posibilidad de su salida pero sólo a través de "otra elección" o que renuncie debido a la presión popular.

Vargas Llosa centra su charla en el fracaso de América latina en sus intentos de desarrollo. Los diversos tópicos o temas son tratados en función de éste su enunciado esencial. El entramado temático que se inscribe en tal Esquema Básico indica que el fracaso latinoamericano sería el producto de la falta de una visión integral y liberal del desarrollo que crea desconfianza en las instituciones, gobiernos corruptos, decepción y genera búsquedas sociales que concluyen en gobiernos como el de Hugo Chávez que empeoran la situación social.

En el caso de Carlos fuentes el Esquema Básico Incluyente se bifurca. Por una parte, se traza una línea argumental que se sintetiza en la salida democrática de 
Encuentro №. 102, 75-90, 2015

un dictador (Chávez) y por otra, una segunda línea argumental que se expresa en la necesidad de cambiar la forma de hacer política, no sólo en el caso de Chávez, sino en el de toda América Latina.

\subsection{Esquemas actanciales}

A través de los esquemas actanciales se observa la representación de los actores sociales en el discurso, además de sus motivaciones, las intenciones que les mueven, los recursos de los cuales se valen y los procesos en los que intervienen.

Estos esquemas se construyen sobre dos ejes: uno horizontal o eje donde se ubican los actantes y otro vertical o eje que da cuenta de la situación del proceso. En el presente trabajo se ha construido un esquema o matriz actancial sólo para los temas en los cuales se menciona de manera directa a Hugo Chávez y a su gobierno y, además, se hallan algunas similitudes o diferencias significativas entre los discursos de los autores tratados.

En el siguiente cuadro (cuadro 2) se observa cómo, según Tomás Eloy Martínez, la pobreza, la educación y la salud deficientes y la corrupción condujeron a que las mayorías eligieran a Chávez presidente. El mismo argumento en el que las mayorías se transforman en agentes expresa Vargas Llosa. Una situación de deterioro en el que priva la corrupción de unas minorías habría conducido a votar por Hugo Chávez, luego la situación se agravaría (cuadro 3).

Cuadro 2. Matriz para el análisis de las zonas actanciales: Tomás Eloy Martínez

\begin{tabular}{|l|l|l|l|l|l|l|}
\hline \multirow{2}{*}{ Temas } & \multicolumn{2}{|l|}{ Zona de la anterioridad } & \multicolumn{2}{l|}{ Zona del evento } & \multicolumn{2}{l|}{ Zona de la posterioridad } \\
\cline { 2 - 7 } & Causa & Instrumento & Agente & Paciente & Destinatario & Finalidad \\
\hline \multirow{2}{*}{$\begin{array}{l}\text { Elección } \\
\text { de Chávez }\end{array}$} & $\begin{array}{l}\text { Pobreza, } \\
\text { educación } \\
\text { y salud } \\
\text { deficiente y } \\
\text { corrupción }\end{array}$ & Voto & $\begin{array}{l}\text { Mayoría } \\
\text { abrumadora }\end{array}$ & $\begin{array}{l}\text { Hugo } \\
\text { Chávez }\end{array}$ & & $\begin{array}{l}\text { Empeora } \\
\text { la } \\
\text { situación }\end{array}$ \\
\hline
\end{tabular}

Es preciso atender con cierta precaución la zona del evento en el caso de Vargas Llosa. A pesar de que aquí aparece Chávez como el paciente de la acción electoral de las mayorías asqueadas, debe matizarse esta situación. La gente asqueada busca y encuentra a alguien que por su condición de demagogo y poseedor de carisma, aunque el autor no lo dice expresamente, tiene la capacidad de manipular (condición de todo demagogo) y de auto-representarse como salvador (carisma). Así que si bien es cierto, los actantes aparecen discursivamente tal como se ha representado en el cuadro 3, no es menos cierto que el uso de los adjetivos demagogo y carisma le asignan a Chávez un poder persuasivo especial sobre esas mayorías que votarán por él. 
Cuadro 3. Matriz para el análisis de las zonas actanciales: Vargas Llosa

\begin{tabular}{|l|l|l|l|l|l|l|}
\hline \multirow{2}{*}{ Temas } & \multicolumn{2}{|l|l|l|}{ Zona de la anterioridad } & \multicolumn{2}{l|}{ Zona del evento } & \multicolumn{2}{l|}{ Zona de la posterioridad } \\
\cline { 2 - 6 } & Causa & Instrumento & Agente & Paciente & Destinatario & $\begin{array}{l}\text { Finalidad o } \\
\text { consecuencia }\end{array}$ \\
\hline $\begin{array}{l}\text { Elección } \\
\text { de Chávez } \\
\text { anteriores } \\
\text { a } 1998 \\
\text { Corruptos } \\
\text { que } \\
\text { enriquecían } \\
\text { a minorías }\end{array}$ & $\begin{array}{l}\text { Voto por un } \\
\text { cambio }\end{array}$ & $\begin{array}{l}\text { Mayorías } \\
\text { asqueadas y } \\
\text { disgustados }\end{array}$ & $\begin{array}{l}\text { Hugo } \\
\text { Chávez }\end{array}$ & & & Destruir al país \\
\hline
\end{tabular}

Puede verse en los cuadros 4 y 5 como Tomás Eloy Martínez y Vargas Llosa concuerdan cuando tratan el tema del mal gobierno de Chávez y la crisis venezolana; establecen que la causa es el gobierno de Chávez y el agente es precisamente el propio Chávez mientras que sobre los venezolanos recae la acción de Chávez.

Cuadro 4. Matriz para el análisis de las zonas actanciales: Tomás Eloy Martínez

\begin{tabular}{|c|c|c|c|c|c|c|}
\hline \multirow[b]{2}{*}{ Temas } & \multicolumn{2}{|c|}{ Zona de la anterioridad } & \multicolumn{2}{|c|}{ Zona del evento } & \multicolumn{2}{|c|}{ Zona de la posterioridad } \\
\hline & Causa & Instrumento & Agente & Paciente & Destinatario & $\begin{array}{l}\text { Finalidad o } \\
\text { consecuencia }\end{array}$ \\
\hline $\begin{array}{l}\text { Mal } \\
\text { Gobierno. } \\
\text { Se agrava } \\
\text { la crisis en } \\
\text { Venezuela }\end{array}$ & $\begin{array}{l}\text { Gobierno } \\
\text { de Chávez }\end{array}$ & $\begin{array}{l}\text { Promesas } \\
\text { incumpli } \\
\text { das. Partido } \\
\text { único, } \\
\text { institucio } \\
\text { nalidad } \\
\text { sometida. }\end{array}$ & Chávez & Venezolanos & & $\begin{array}{l}\text { El país está } \\
\text { peor que antes }\end{array}$ \\
\hline
\end{tabular}

Cuadro 5. Matriz para el análisis de las zonas actanciales: Vargas Llosa

\begin{tabular}{|l|l|l|l|l|l|l|}
\hline \multirow{2}{*}{ Temas } & \multicolumn{2}{|l|}{ Zona de la anterioridad } & \multicolumn{2}{l|}{ Zona del evento } & \multicolumn{2}{l|}{ Zona de la posterioridad } \\
\cline { 2 - 7 } & Causa & Instrumento & Agente & Paciente & Destinatario & Finalidad \\
\hline $\begin{array}{l}\text { Chávez } \\
\text { ejemplo } \\
\text { del fracaso } \\
\text { latinoameri } \\
\text { cano. Mal } \\
\text { gobierno }\end{array}$ & Gobierno de & $\begin{array}{l}\text { Demagogia } \\
\text { gangsterismo, } \\
\text { Chictadura }\end{array}$ & Chávez & Venezolanos & & $\begin{array}{l}\text { El país se } \\
\text { debate en } \\
\text { una crisis } \\
\text { atroz }\end{array}$ \\
\hline
\end{tabular}

Cuando Martínez y Fuentes tratan las acciones que debe llevar a cabo la mayoría de la población frente al gobierno de Hugo Chávez (Cuadros 6 y 7 ) recurren a la democracia, al mecanismo del voto. Es importante anotar aquí algunas diferencias entre los autores. Por una parte, Tomás Eloy Martínez acepta que se realicen otras 
elecciones o que renuncie Chávez a la presidencia pero llama la atención acerca del peligro de acelerar los tiempos democráticos y por otra, Carlos Fuentes introduce la posible ayuda de Brasil y México en la solución de la crisis venezolana.

Cuadro 6. Matriz para el análisis de las zonas actanciales: Tomás Eloy Martínez

\begin{tabular}{|l|l|l|l|l|l|l|}
\hline \multirow{2}{*}{ Temas } & \multicolumn{2}{|c|}{ Zona de la Anterioridad } & \multicolumn{2}{c|}{ Zona del Evento } & \multicolumn{2}{c|}{ Zona de la Posterioridad } \\
\cline { 2 - 7 } & Causa & Instrumento & Agente & Paciente & Destinatario & Finalidad \\
\hline $\begin{array}{l}\text { Salida } \\
\text { democrática }\end{array}$ & $\begin{array}{l}\text { Gobierno } \\
\text { de Chávez }\end{array}$ & $\begin{array}{l}\text { Elecciones } \\
\text { o renuncia } \\
\text { sin violentar } \\
\text { los tiempos } \\
\text { democráticos }\end{array}$ & $\begin{array}{l}\text { Venezola- } \\
\text { nos }\end{array}$ & $\begin{array}{l}\text { Gobierno } \\
\text { de Hugo } \\
\text { Chávez }\end{array}$ & Venezolanos & $\begin{array}{l}\text { Salir de } \\
\text { un mal } \\
\text { gobierno }\end{array}$ \\
\hline
\end{tabular}

Cuadro 7. Matriz para el análisis de las zonas actanciales: Carlos Fuentes

\begin{tabular}{|c|c|c|c|c|c|c|}
\hline \multirow{2}{*}{ Temas } & \multicolumn{2}{|c|}{ Zona de la Anterioridad } & \multicolumn{2}{|c|}{ Zona del Evento } & \multicolumn{2}{|c|}{ Zona de la Posterioridad } \\
\hline & Causa & Instrumento & Agente & Paciente & Destinatario & Finalidad \\
\hline \begin{tabular}{|l} 
Salida \\
democrática, \\
venezolana \\
y con ayuda \\
latinoameri- \\
cana
\end{tabular} & $\begin{array}{l}\text { Gobierno } \\
\text { de Chávez }\end{array}$ & $\begin{array}{l}\text { Elecciones, } \\
\text { plebiscito o } \\
\text { aplicación } \\
\text { de carta } \\
\text { democrática }\end{array}$ & $\begin{array}{l}\text { Venezola- } \\
\text { nos o } \\
\text { México y } \\
\text { Brasil }\end{array}$ & $\begin{array}{l}\text { Gobierno } \\
\text { de Hugo } \\
\text { Chávez }\end{array}$ & Venezolanos & $\begin{array}{l}\text { Salir de una } \\
\text { dictadura }\end{array}$ \\
\hline
\end{tabular}

\subsection{Perspectiva desde la cual se construye la imagen de Hugo Chávez y su gobierno}

En el análisis actancial se debe contemplar la perspectiva bajo la cual los autores presentan el proceso conceptualizado (Molero, 2003). Es decir, la construcción del evento desde la perspectiva del yo o desde la perspectiva del otro. Se ha integrado en el cuadro 8, la conceptualización de Hugo Chávez y de su gobierno desde la perspectiva del yo de cada uno de los autores, de forma tal de establecer una apreciación integral que parta de la comparación del discurso de los tres autores.

En primer lugar, se observa cómo los tres novelistas son partícipes de una visión negativa de lo que acontecía en Venezuela en cada uno de los momentos en que fueron expresados los textos analizados. En segundo lugar, para los tres, la causa de tal degradación es el presidente Hugo Chávez. En tercer lugar, se destaca una diferencia importante entre la posición de duda e incertidumbre de Tomás Eloy Martínez frente a sacar del gobierno a Chávez y la de los otros dos autores. Para este escritor, aunque acepta otra elección o la renuncia del presidente, es necesario respetar los tiempos democráticos debido a los peligros que su violación podría acarrear. Vargas Llosa no entra a detallar aspectos de la crítica situación que vivía Venezuela, sino que expresa el desideratum, de carácter general, con lo que incluye a todos los países latinoamericanos, de llevar "a la política gentes decentes, gentes que no roben, gentes que hagan lo que dicen que van a hacer, que no mientan o que mientan poco, lo inevitable"; tal generalidad permite que se pueda aplicar al caso 
venezolano. Y Carlos Fuentes, al igual que Vargas Llosa, ofrece una posibilidad de mejoramiento de la situación, pero esta vez sí se propone abiertamente la salida de Chávez del gobierno. En cuarto lugar los tres expresan su adhesión a la democracia, en el caso de Fuentes es explícita la referencia al caso venezolano: Chávez debe salir por métodos democráticos. La aprehensión de Martínez acerca de las bondades de esa posibilidad se deben precisamente a su apego a los tiempos que exige la democracia y Vargas Llosa durante todo el desarrollo de la charla habla de una sociedad democrática en la que las instituciones adquieran credibilidad y cuando aspira al adecentamiento de la política se entiende que es democráticamente, dice textualmente este autor cuando hace referencia al caso del Chile de Pinochet "las dictaduras no han sido jamás una solución para los problemas latinoamericanos... han hecho de la corrupción una forma natural de gobierno...."

Cuadro 8. Conceptualización de eventos: Hugo Chávez y su gobierno.

Cuadro comparativo

\begin{tabular}{|c|c|c|c|}
\hline & Tomás Eloy Martínez & Vargas Llosa & Carlos Fuentes \\
\hline $\begin{array}{l}\text { Visión de } \\
\text { la situación } \\
\text { actual }\end{array}$ & $\begin{array}{l}\text { Negativa de degradación: } \\
\text { Chávez ha tratado de "forjar } \\
\text { una nación regida por un } \\
\text { partido único y de someter } \\
\text { a todas las instituciones a su } \\
\text { voluntad" } \\
\text { "El país está peor que antes..." }\end{array}$ & $\begin{array}{l}\text { Negativa de } \\
\text { degradación: Un } \\
\text { gran demagogo, } \\
\text { carismático, } \\
\text { gangster y bandido } \\
\text { que puede destruir } \\
\text { a Venezuela. } \\
\text { "Venezuela se } \\
\text { debate en una crisis } \\
\text { atroz" }\end{array}$ & $\begin{array}{l}\text { Negativa de } \\
\text { degradación: } \\
\text { "Venezuela parece } \\
\text { tener un presidente } \\
\text { decidido a llevar } \\
\text { al desastre al país } \\
\text { y a su gobierno, es } \\
\text { decir morir en la } \\
\text { hoguera, él mismo, } \\
\text { Chávez, y todo su } \\
\text { gobierno" } \\
\text { Un demagogo, } \\
\text { payaso y dictador } \\
\text { en el poder. }\end{array}$ \\
\hline $\begin{array}{l}\text { Causantes } \\
\text { de la } \\
\text { degradación }\end{array}$ & Hugo Chávez & Hugo Chávez & Hugo Chávez \\
\hline $\begin{array}{l}\text { Tipo de } \\
\text { cambio } \\
\text { propuesto } \\
\text { por el autor }\end{array}$ & $\begin{array}{l}\text { Incertidumbre: Venezuela, no } \\
\text { sabe cómo sacarse de encima } \\
\text { al presidente Hugo Chávez. } \\
\text { "Si Chávez destruyó casi todas } \\
\text { las instituciones que estaban } \\
\text { antes de su llegada., ¿tiene } \\
\text { ya Venezuela con qué llenar } \\
\text { ese vacío cuando desaparezca } \\
\text { Chávez?" } \\
\text { Sacarse a Chávez, pero acelerar } \\
\text { los tiempos de la democracia } \\
\text { puede crear remedios peores } \\
\text { que la enfermedad }\end{array}$ & $\begin{array}{l}\text { De mejoramiento: } \\
\text { 1.- Integrar } \\
\text { desarrollo } \\
\text { económico, político } \\
\text { y cultural. } \\
\text { 2.- Llevar gente } \\
\text { decente al } \\
\text { Gobierno. } \\
\text { 3.- Emprender } \\
\text { reformas liberales y } \\
\text { hacerlas bien. }\end{array}$ & $\begin{array}{l}\text { De mejoramiento: } \\
\text { Salir de Chávez e } \\
\text { iniciar "un proceso } \\
\text { de renovación } \\
\text { democrática" }\end{array}$ \\
\hline
\end{tabular}




\begin{tabular}{|l|l|l|l|}
\hline $\begin{array}{l}\text { Los agentes } \\
\text { que pueden } \\
\text { cambiar lo } \\
\text { actual }\end{array}$ & Los venezolanos & $\begin{array}{l}\text { Venezolanos, } \\
\text { gobernantes que } \\
\text { tengan un concepto } \\
\text { integral del } \\
\text { desarrollo, liberales } \\
\text { y gente decente. }\end{array}$ & $\begin{array}{l}\text { Venezolanos y } \\
\text { Brasil y México }\end{array}$ \\
\hline $\begin{array}{l}\text { Instru } \\
\text { mentos del } \\
\text { cambio }\end{array}$ & $\begin{array}{l}\text { El voto o la renuncia, aunque } \\
\text { salir de Chávez, puede ser } \\
\text { peligroso. }\end{array}$ & $\begin{array}{l}\text { Llevar gente decente } \\
\text { al gobierno, con } \\
\text { una visión integral } \\
\text { del desarrollo y } \\
\text { emprenda reformas } \\
\text { liberales. }\end{array}$ & $\begin{array}{l}\text { Un plebiscito, } \\
\text { una elección o la } \\
\text { aplicación de la } \\
\text { Carta Democrática. }\end{array}$ \\
\hline
\end{tabular}

\section{Nivel lingüístico}

Todo lo dicho arriba constituye la base para el análisis lingüístico. Este nivel de análisis permite observar cómo son presentados los núcleos de sentido y las relaciones que se establecen entre ellos. En esta parte se consideran solamente los dominios lingüísticos y los campos semánticos.

\subsection{Dominios}

Los dominios lingüísticos se refieren a dominios socio-experienciales que "permiten identificar las prácticas sociales y discursivas a las cuales alude el emisor para encontrar el marco adecuado que le permita obtener los significados que desea atribuir a las palabras clave de sus mensajes" (Molero, 2003).

Los documentos analizados son ricos en argumentos y abundantes en matices discursivos que tocan diversos aspectos y que de una u otra forma se refieren a la realidad venezolana, es por ello que, aún siendo evidente el dominio político (aunque no el único) desde el cual se emiten las opiniones de cada uno de los autores, para los fines comparativos, se han configurado sub-dominios en cinco ámbitos particulares de ese dominio político. En primer lugar, la forma de gobierno o régimen de gobierno, en segundo lugar, Hugo Chávez y las características que lo definen como gobernante; en tercer lugar, la acción gubernamental, en cuarto lugar, el origen del gobierno y, en quinto y último lugar, la forma de lucha que proponen contra el gobierno.

A diferencia de Martínez y de Fuentes, Vargas Llosa en su charla no hace mención de la forma como se organizan los poderes públicos en el gobierno de Chávez (cuadro 9). Martínez llama a la forma de gobierno democracia autoritaria, mientras que Fuentes, sin llegar a afirmar que existe una dictadura, dice que Chávez es un dictador de allí que se pueda inferir que la forma que adopta el gobierno sea dictatorial. Cuando se refieren a Hugo Chávez (cuadro 10) como gobernante los calificativos abundan. Lo significativo es la cualidad negativa de los mismos; no es posible encontrar un solo término o frase que exprese algo positivo del gobernante venezolano. 
Cuadro 9. Sub dominio: forma de gobierno

Cuadro compartivo

\begin{tabular}{|c|c|c|}
\hline Tomás Eloy Martínez & Mario Vargas Llosa & Carlos Fuentes \\
\hline Textos & Textos & Textos \\
\hline $\begin{array}{l}\text { - Trata de "forjar una } \\
\text { nación regida por } \\
\text { un partido único } \\
\text { y de someter todas } \\
\text { las instituciones a su } \\
\text { voluntad" } \\
\text { "como sucede con los } \\
\text { regímenes autoritarios, se } \\
\text { confundían los intereses } \\
\text { del Gobierno de turno } \\
\text { con los intereses de la } \\
\text { nación" } \\
\text { Democracia autoritaria }\end{array}$ & & $\begin{array}{l}\text { - Chávez "actúa como los } \\
\text { dictadores, como los } \\
\text { políticos autoritarios, y él } \\
\text { es uno de ellos." } \\
\text { - Pone en "entredicho } \\
\text { la vida y la libertad de } \\
\text { muchos" } \\
\text { "Amenazando a } \\
\text { personas" }\end{array}$ \\
\hline
\end{tabular}

Cuadro 10. Subdominio: Hugo Chávez, el gobernante

Cuadro comparativo

\begin{tabular}{|c|c|c|}
\hline Tomás Eloy Martínez & Mario Vargas Llosa & Carlos Fuentes \\
\hline Textos & Textos & Textos \\
\hline $\begin{array}{l}\text { - } \quad \text { No tiene talento: } \\
\text { "aunque estaba claro } \\
\text { desde el principio } \\
\text { que Chávez no tenía } \\
\text { el talento..." } \\
\text { - No tiene "decisión } \\
\text { arrolladora que hacen } \\
\text { falta para...." } \\
\text { - Se convirtió "durante } \\
\text { sus dos años de } \\
\text { cárcel, en un Mesías } \\
\text { irrefutable" }\end{array}$ & $\begin{array}{l}\text { - "Este país (Venezuela)....tiene al } \\
\text { frente del gobierno a un gran } \\
\text { demagogo. } \\
\text { "Paulo Rabello de Brasil decía } \\
\text { que las gentes que han votado } \\
\text { por millones, por Lula, no han } \\
\text { votado por el socialismo en } \\
\text { la mayoría de los casos, han } \\
\text { votado por algo diferente a lo } \\
\text { que tienen y eso diferente lo ha } \\
\text { conseguido encarnar a través } \\
\text { de carisma o demagogia. Es } \\
\text { lo mismo que ha pasado por } \\
\text { ejemplo, en Venezuela" } \\
\text { “...Chávez (Venezuela)... } \\
\text { verdaderos gángsteres, } \\
\text { auténticos bandidos que entran } \\
\text { al gobierno como entra un } \\
\text { ladrón a una casa a robar a } \\
\text { saquear, a enriquecerse de la } \\
\text { manera más cínica, más rápida } \\
\text { posible." }\end{array}$ & $\begin{array}{l}\text { - Chávez es un } \\
\text { hombre sin } \\
\text { ninguna seriedad" } \\
\text { - "Es un demagogo" } \\
\text { - "Un payaso" } \\
\text { "Está siempre } \\
\text { en escena } \\
\text { invocando frases } \\
\text { grandilocuentes } \\
\text { sin ninguna base } \\
\text { real de gobierno" } \\
\text { "un político } \\
\text { autoritario" }\end{array}$ \\
\hline
\end{tabular}


Encuentro №. 102, 75-90, 2015

Idénticas son las expresiones en torno al ejercicio de su gobierno. En los tres autores está presente una idea negativa del ejercicio gubernamental, las expresiones definen lo que se podría denominar un mal gobierno y, obviamente, unas consecuencias de ese mal gobierno muy perjudiciales para el país (véase cuadro 11).

Cuadro 11. Sub dominio: ejercicio de gobierno

Cuadro comparativo

\begin{tabular}{|c|c|c|}
\hline Tomás Eloy Martínez & Mario Vargas Llosa & Carlos Fuentes \\
\hline Textos & Textos & Textos \\
\hline $\begin{array}{l}\text { - } \quad \text { "La popularidad de } \\
\text { Chávez descendió al } \\
\text { mismo ritmo que el } \\
\text { valor del petróleo" } \\
\text { "No se sabe cómo } \\
\text { administró el } \\
\text { teniente coronel } \\
\text { la inverosímil } \\
\text { riqueza que entró } \\
\text { a Venezuela en los } \\
\text { últimos dos años, } \\
\text { sin que ninguna } \\
\text { de sus grandes } \\
\text { promesas se haya } \\
\text { cumplido". } \\
\text { "El país está peor } \\
\text { que antes" } \\
\text { "El espejismo de } \\
\text { Chávez se evapora" } \\
\text { "Destruyó casi todas } \\
\text { las instituciones que } \\
\text { estaban antes de su } \\
\text { llegada" }\end{array}$ & 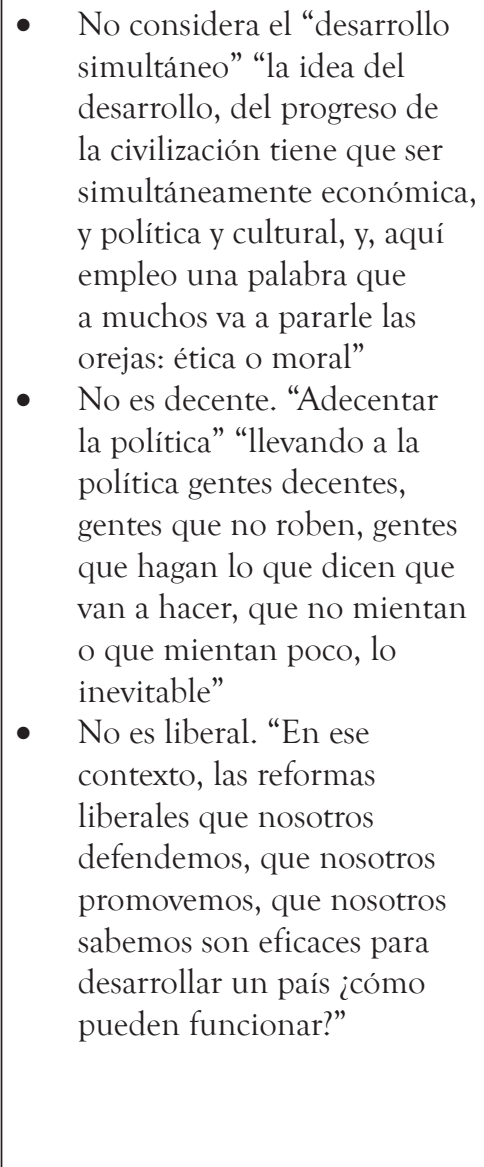 & $\begin{array}{l}\text { - "Un gobierno debe } \\
\text { propiciar todo lo } \\
\text { contrario de lo que } \\
\text { ha hecho Chávez } \\
\text { para desgracia de } \\
\text { Venezuela" } \\
\text { - “...menos } \\
\text { dependencia del } \\
\text { exterior" } \\
\text { - “...confianza en } \\
\text { la base social, } \\
\text { en el capital } \\
\text { humano" } \\
\text { "...construir } \\
\text { desde abajo hacia } \\
\text { arriba" } \\
\text { "...no estar } \\
\text { dependiendo } \\
\text { de que los } \\
\text { problemas sólo } \\
\text { se resuelvan en } \\
\text { la cima de la } \\
\text { pirámide" } \\
\text { Ha creado un } \\
\text { constante "clima de } \\
\text { peligro, amenaza, } \\
\text { sobresalto, } \\
\text { incertidumbre...” }\end{array}$ \\
\hline
\end{tabular}

Chávez llegó a la presidencia democráticamente (cuadro 12) y democráticamente debe salir del gobierno (cuadro 13), esa es la idea que expresan los autores cuando tratan los temas del origen del gobierno de Chávez y la forma de lucha que deben adoptar los venezolanos. 
Cuadro 12. Sub dominio: origen del gobierno

Cuadro comparativo

\begin{tabular}{|l|l|l|}
\hline Tomás Eloy Martínez & Mario Vargas Llosa & Carlos Fuentes \\
\hline Textos & Textos & Textos \\
\hline - "Chávez fue elegido por & $\bullet \quad$ "El ha llegado al poder & $\bullet$ "llegó \\
democráticamente \\
una mayoría abrumadora" & con el voto de una gran & a la presidencia" \\
al elegir a Chávez, pero & totalmente disgustados & \\
esa elección se ratificó al & y asqueados de la & \\
menos dos veces..." & democracia que tenían..." & \\
\hline
\end{tabular}

Una posible salida militar es negada de plano. Debe recordarse que para la fecha que escribe Tomás Eloy Martínez (febrero) ya se habían producido dos pronunciamientos de militares activos en contra de Chávez y ante estos hechos fija posición y los condena expresando lo siguiente: "Lo que proponen Soto y Flores (los dos militares) es el mismo remedio que Chávez quiso aplicar hace diez años ${ }^{6} \mathrm{y}$ que produjo, junto al derrumbe estrepitoso de la vieja clase política, la aparición de una democracia autoritaria.." y más adelante dice que "si en el camino se mezclaran pronunciamientos militares, lo único que lograrían es enturbiar un proceso ya de por sí traumático.”

Carlos Fuentes es tan tajante como Martínez. Un golpe militar "sería un retroceso que nos arrojaría a ese abismo del pasado latinoamericano". Por último, Vargas Llosa expresa su desprecio por las dictaduras militares, dice que "han hecho de la corrupción una forma natural de gobierno".

Cuadro 13. Sub dominio: forma de lucha contra Chávez

Cuadro comparativo

\begin{tabular}{|c|c|c|}
\hline Tomás Eloy Martínez & Mario Vargas Llosa & Carlos Fuentes \\
\hline Textos & Textos & Textos \\
\hline $\begin{array}{l}\text { - La única manera } \\
\text { aceptable de } \\
\text { enmendar ahora } \\
\text { aquel error es } \\
\text { otra elección. } \\
\text { A menos que, } \\
\text { por efecto de } \\
\text { una masiva } \\
\text { presión popular, } \\
\text { el presidente } \\
\text { accediera a } \\
\text { renunciar..." }\end{array}$ & $\begin{array}{l}\text { - } \quad \text { "La cultura defiende contra la } \\
\text { demagogia, defiende contra la } \\
\text { equivocación terrible de elegir mal } \\
\text { en unas elecciones" } \\
\text { - La cultura "ayuda a crear esos } \\
\text { consensos que han permitido por } \\
\text { ejemplo los casos muchas veces } \\
\text { ejemplares de España y de Chile" } \\
\text { El liberalismo es una tradición } \\
\text { que hay que defender no solo por } \\
\text { homenaje a la verdad, sino porque } \\
\text { vivimos un momento difícil de la } \\
\text { historia en la que ese progreso y esa } \\
\text { civilización están amenazados" }\end{array}$ & $\begin{array}{l}\text { - "debe salir también } \\
\text { democráticamente, } \\
\text { sea a través de un } \\
\text { plebiscito, una } \\
\text { elección o por } \\
\text { la aplicación.... } \\
\text { de la Carta } \\
\text { Democrática" }\end{array}$ \\
\hline
\end{tabular}

6 Se refiere al golpe militar fallido que lideró Hugo Chávez el 4 de febrero de 1992. 


\subsection{Campos semánticos}

Los campos semánticos o léxicos se refieren al conjunto de términos (lexías) que poseen un rasgo mínimo de significado común o sema. Los términos que utilizan los novelistas para construir la imagen de Hugo Chávez son todos degradantes. La representación que realizan del mandatario es negativa (cuadro 14). Especialmente severos en los calificativos son Vargas Llosa y Fuentes. Otro rasgo común a los autores es que se abstienen del uso de lexías complejas en tal representación. Excepto el "muchachote" y "confuso delta" de Martínez las restantes son de una simplicidad expresiva desconcertante.

Cuadro 14. Campos semánticos: construcción del concepto Hugo Chávez

Cuadro comparativo

\begin{tabular}{|c|c|c|c|}
\hline $\begin{array}{l}\text { Campo } \\
\text { semántico }\end{array}$ & Tomás Eloy Martínez & Mario Vargas Llosa & Carlos Fuentes \\
\hline Hugo Chávez & $\begin{array}{ll}\text { - } & \text { Sin talento } \\
\text { - } & \text { Sin decisión } \\
\text { arrolladora } \\
\text { - } \\
\text { Muchachote al que } \\
\text { Venezuela le queda } \\
\text { grande } \\
\text { - Teniente Coronel } \\
\text { - Es el confuso } \\
\text { delta en el que } \\
\text { desembocó } \\
\text { Venezuela }\end{array}$ & $\begin{array}{ll}\text { - } & \text { Gran demagogo } \\
\text { - } & \text { Gángster } \\
\text { - } & \text { Bandido }\end{array}$ & $\begin{array}{ll}\text { - } & \text { Demagogo } \\
\text { - } & \text { Payaso } \\
\text { - } & \text { Sin ninguna } \\
& \text { seriedad } \\
\text { - } & \text { Actúa como los } \\
& \text { dictadores, es uno } \\
& \text { de ellos } \\
\text { - } & \text { Político } \\
& \text { autoritario }\end{array}$ \\
\hline
\end{tabular}

\subsection{Organización del discurso}

Los campos léxicos se asocian a los modos como se organiza el discurso. Describen, narran, argumentan de acuerdo a la intencionalidad del discurso; estos diferentes modos tienden articularse de diversas formas en el discurso. Dado que el discurso político trata básicamente de convencer o persuadir a unos receptores determinados de allí su carácter polémico, de contraposición, tiende a ser predominantemente argumentativo.

Todo discurso argumentativo se expresa de la siguiente forma: una aserción de partida, una aserción de pasaje para concluir en la de llegada. Así se han construido los cuadros que a continuación se presentan. Aunque en un discurso pueda hallarse una cantidad importante de expresiones argumentativas, se ha reducido al máximo las mismas al intentar reconstruir aquellos argumentos centrales del discurso de cada uno de los autores.

En el caso de Tomás Eloy Martínez el argumento es básicamente el que se presenta en el cuadro 15. Habría que complementar la aserción de llegada con la tesis de que aún con unas nuevas elecciones o la renuncia del presidente, el hecho de adelantar los tiempos democráticos podría ser peligroso para el país. 
Cuadro 15. Modo de organización del discurso: relación argumentativa, Tomás Eloy Martínez

\begin{tabular}{|l|l|l|}
\hline Aserción de partida & Aserción de pasaje & Aserción de llegada \\
\hline $\begin{array}{l}\text { Chávez fue elegido } \\
\text { por una mayoría } \\
\text { abrumadora }\end{array}$ & $\bullet \quad \begin{array}{l}\text { Chávez ha hecho un } \\
\text { mal gobierno }\end{array}$ & $\begin{array}{l}\text { Venezuela no sabe cómo } \\
\text { sacarse de encima al } \\
\text { presidente }\end{array}$ \\
& $\begin{array}{l}\text { Sólo es aceptable una } \\
\text { nueva elección o la } \\
\text { renuncia del presidente }\end{array}$ \\
\hline
\end{tabular}

Fíjese como la argumentación central del texto de Fuentes es esencialmente la misma de Tomás Eloy Martínez. Los aspectos que la diferencian son menores, entre otros, los calificativos con los que Fuentes representa a Chávez y la idea de la aplicación de la Carta Democrática.

Cuadro 16. Modo de organización del discurso: relación argumentativa, Carlos Fuentes

\begin{tabular}{|c|c|c|}
\hline Aserción de partida & Aserción de pasaje & Aserción de llegada \\
\hline $\begin{array}{l}\text { - Chávez llegó } \\
\text { democráticamente a la } \\
\text { Presidencia }\end{array}$ & $\begin{array}{l}\text { Chávez es un payaso, } \\
\text { demagogo, dictador, } \\
\text { autoritario } \\
\text { Chávez lleva a } \\
\text { Venezuela al desastre }\end{array}$ & $\begin{array}{l}\text { Debe salir } \\
\text { democráticamente: } \\
\text { elecciones, plebiscito, } \\
\text { aplicación de carta } \\
\text { democrática. }\end{array}$ \\
\hline
\end{tabular}

El texto de Vargas Llosa es diferente al de los otros dos autores. La argumentación central del texto es que América Latina ha fracasado en sus intentos de desarrollo debido a que no posee un concepto integral del desarrollo. La inclusión de Hugo Chávez y de su gobierno así como la de Fujimori y la de otros gobernantes o ex-gobernantes latinoamericanos es primordialmente ejemplificadora de ese fracaso.

Para el caso estrictamente venezolano la relación argumentativa podría presentarse tal como se hace en el cuadro 17. Puede notarse que la argumentación es bastante cercana a la de los otros autores, sin embargo, la aserción de llegada se diferencia debido a que el evento central de su disertación no es Chávez y su gobierno y la forma de actuar frente a la crisis, sino el subdesarrollo latinoamericano en general.

Cuadro 17. Modo de organización del discurso: relación argumentativa, Vargas Llosa

\begin{tabular}{|l|l|l|}
\hline Aserción de partida & Aserción de pasaje & Aserción de llegada \\
\hline - $\quad$ La gente asqueada & $\bullet \quad \begin{array}{l}\text { Chávez es un gran } \\
\text { demagogo y un } \\
\text { búsquedada en la algo } \\
\text { diferente votó por } \\
\text { Chávez }\end{array}$ & $\bullet \begin{array}{l}\text { Venezuela se debate en } \\
\text { una crisis atroz. } \\
\text { Puede destruir a } \\
\text { Venezuela }\end{array}$ \\
\hline
\end{tabular}




\section{Nota final: la intención de los autores}

Todo discurso tiene una intención comunicativa; alguna razón posee el emisor del mensaje para producirlo. Debido a que esta intención no siempre se encuentra explícita en los textos, como efectivamente no lo está en los analizados en este trabajo, debemos recurrir a lo dicho hasta el momento para tratar de desentrañarla. Es posible concluir que en el caso de Tomás Eloy Martínez se halla básicamente el propósito de advertir acerca de los peligros de saltarse los tiempos democráticos, ya sea a través de un pronunciamiento militar, o de unas elecciones o de la propia renuncia presidencial.

Nos parece que la intención de Vargas Llosa es fundamentalmente ejemplificadora del fracaso latinoamericano. Así como menciona a Hugo Chávez, lo hace también con Fujimori de Perú, con Lula de Brasil o con Alemán de Nicaragua. Estos gobernantes representan la corrupción y la demagogia de algunos gobernantes latinoamericanos.

Por último, la declaración de Fuentes pudiera tener dos propósitos. Uno, advertir sobre los peligros de un golpe militar y, segundo, justificar la necesidad de salir de Hugo Chávez.

\section{Referencias bibliográficas}

Cabeza, J. \& Molero, L. (2005). Semiotización e interpretación discursivas. El caso del "llanto" por la muerte de Ignacio Sánchez Mejías de Federico García Lorca y el de la "elegía" por la muerte de Tomás Alfaro Calatrava de Benito Raúl Losada. Revista Lingua Americana. (15). Instituto de Investigaciones Literarias y Lingüísticas de la Facultad de Humanidades y Educación de la Universidad del Zulia.

Fuentes, C. (2002, 14 de diciembre). Carlos Fuentes no aboga por una salida militar. El Nacional. Recuperado el 10 de diciembre de 2004, de www. Forumfrancevenezuela.com.ve/pri_04es.html

Martínez, T. E. (2002, 16 de febrero). La soga al cuello. Diario La Nación. p. 19.

Molero, L. (1985). Lingüística y discurso. Maracaibo: Facultad Experimental de Ciencias. Universidad del Zulia.

Molero, L. (1999). Análisis de dos discursos del proceso electoral de 1998 bajo un enfoque semántico pragmático. En Bolívar y Kohn (Comp). El discurso político venezolano. Un estudio multidisciplinario. (pp. 145 - 157). Caracas: Tropykos.

Molero, L. (2002). El personalismo en el discurso político venezolano. Un enfoque semántico-pragmático. Revista Espacio Abierto, 2 (2), 291-334.

Molero, L. (2003). El enfoque semántico-pragmático en el análisis del discurso. Visión teórica actual. Lingua Americana, VII (12), 5 - 28.

van Dijk, T. A. (comp). (1977). El discurso como interacción social. Barcelona: Gedisa. Vargas Llosa, M. (2003). ¿Por qué fracasa América Latina?. Inter-Forum, Recuperado el 15 de diciembre de 2004, de www.revistainterforum.com/espanol/ articulos/021203soc_fracasa_llosa.html http://www.revistainterform.com/ espanol/articulos/021203soc_fracasa_llosa.html 